\title{
EL CASO CAMPANELLI Y PARADISO ANTE EL TRIBUNAL EUROPEO DE DERECHOS HUMANOS: EL CONCEPTO DE FAMILIA DE FACTO Y SU APORTACIÓN AL DEBATE DE LA GESTACIÓN POR SUSTITUCIÓN
}

\author{
(CAMPANELLI AND PARADISE VS. ITALY CASE BEFORE \\ THE EUROPEAN COURT OF HUMAN RIGHTS: FAMILY \\ DE FACTO CONCEPT AND ITS CONTRIBUTION \\ TO THE SURROGACY)
}

\author{
Dr. Anna María Ruiz Martín \\ Abogada del ICAM \\ Lecturer in Law \\ ORCID ID: 0000-0001-5020-582X
}

Recibido: 11.06.2019 / Aceptado: 05.07.2019

DOI: https://doi.org/10.20318/cdt.2019.5020

\begin{abstract}
Resumen: En el año 2017, la Gran Sala del Tribunal Europeo de Derechos Humanos (TEDH) se volvió a pronunciar sobre el caso Paradiso y Campanelli c. Italia, dos años después que la Sección $2^{\circ}$ dictase su primera resolución en el caso. Esta sentencia tiene una especial aportación, teniendo en cuenta que el caso no ha sido considerado como un supuesto de gestación por sustitución propio. Sin embargo, se considera que lo que en realidad está aportando esta sentencia y por ello es analizada, es la reflexión que realiza el TEDH sobre la determinación y valoración del vínculo familiar de facto en este tipo de supuestos de gestación por sustitución internacionales, a la luz del artículo 8 del Convenio Europeo de Derechos Humanos (CEDH).
\end{abstract}

Palabras clave: artículo $8 \mathrm{CEDH}$, interés superior del menor, valoración del vínculo familiar, vida familiar de facto, respeto a la vida privada, injerencia del Estado, gestación por sustitución.

\begin{abstract}
In 2017, the Grand Chamber of the European Court of Human Rights (ECHR) rendered its second ruling with regard to the case Paradiso and Campanelly vs. Italy, two years after the first ruling was rendered in 2015. This contribution is aimed to highlight the most remarkable statements of this second Judgment taking into account that this case was never considered a case of surrogacy as such. The moot point relies on the scope and implementation of the Article 8 of the Convention on the Protection of Human Rights and Fundamental Freedoms and the assessment of a real familiar de facto bond in cross border surrogacy cases, given by the judges in this judgment.
\end{abstract}

Keywords: Article 8 CPHFR, best interest of the child, family bonds, family life de facto, right to respect the private life, State interference, public policy, surrogacy.

Sumario: I. Introducción; II. Referencias sobre el supuesto de hecho; III. Las aportaciones del Tribunal Europeo de Derechos Humanos con el caso al debate de la gestación por sustitución: el por qué del giro en su jurisprudencia: 1. El Derecho al respeto a la vida privada y el Derecho a la vida familiar: el Artículo 8 de la Convención Europea de Derechos Humanos: 2. Determinación de la duración del vínculo familiar de facto y el concepto de familia en el Derecho europeo; A) Qué debe entenderse como una familia de facto según el artículo 8 del CEDH; B. Qué no se entiende 
como una familia de facto según la jurisprudencia del artículo 8 del CEDH. Relación con el derecho a la vida privada: IV. Incidencia del pronunciamiento en el Derecho doméstico de los Estados parte de la Convención Europea de Derechos Humanos: 1. El orden público internacional (de protección) en el Estado de destino: la opinión del Tribunal Europeo de Derechos Humanos en el caso: 2. Aportaciones de la Opinión particular de los jueces del caso: V. Conclusiones

\section{Introducción}

1. El presente artículo analiza los aspectos más destacados de una de las sentencias más importantes hasta ahora dictadas por el Tribunal Europeo de Derecho Humanos (en lo sucesivo, TEDH), en el debate de la gestación por sustitución: el asunto Campanelli y Paradiso c. Italia (en adelante, "el caso") ${ }^{1}$. El caso es muy particular, en atención a que no se ha llegado a considerar como un supuesto de gestación por sustitución puro, a diferencia de sus coetáneos, como los asuntos Menesson-Labasse (con los que se suele comparar) ${ }^{2}$. Del mismo además, existen dos resoluciones del TEDH. La primera fue dictada en el año 2015 por la Sección $2^{\text {a }}$ del TEDH. La segunda fue dictada en el año 2017 por la Gran Sala, siendo esta última la definitiva en el caso.

2. El análisis está centrado sobre las cuestiones relativas al ámbito sustantivo de la norma analizada por el TEDH, que es el artículo 8 de la CEDH. No se hace un análisis exhausto de los aspectos conflictivos del ámbito del Derecho internacional privado (DIPr). No obstante, será inevitable tener que realizar al final unas puntualizaciones relativas a los problemas que surgen en estos supuestos en el ámbito del DIPr, por dos razones. Primero: porque la gran parte de los supuestos de gestación por sustitución (y de adopción) atendiendo al variado panorama legislativo en los Estados parte del CEDH son, en su mayoría (por no decir todos), transfronterizos y deber ser solucionados mediante las normas de DIPr, -especialmente de reconocimiento-, de los Estados de destino. Todo ello, en defecto que no existe todavía ninguna norma ni de corte convencional ni europea que haya regulado la situación como bien se sabe ${ }^{3}$. Segundo: como consecuencia de lo anterior, el mismo TEDH observa que las cuestiones relativas al DIPr son necesarias, dado que la gran parte de los nacidos por esta técnica de reproducción, acaban siendo trasladados a un Estado diferente del que han nacido y donde usualmente, la técnica está prohibida ${ }^{4}$. Por ello, los padres comitentes o padres de intención (intended parents) se enfrentan generalmente, a la posible denegación del reconocimiento de la filiación adquirida en el Estado de origen, donde se celebró el contrato de gestación por sustitución y fueron inscritos como los padres del menor de forma legal ${ }^{5}$.

Esta precisión la concibe el TEDH, en cuanto, considera que los Estados parte en los que está prohibida o no regulada la técnica de la gestación por sustitución, no usen el resorte "automático e indiscriminado" al orden público internacional por encima de la protección debida al principio supranacional del "interés superior del menor", para no dar efectos de forma automática mediante el reconocimiento

\footnotetext{
${ }^{1}$ STEDH 24 de enero 2017, $\mathrm{n}^{\mathrm{o}}$ 25358/12, as. Campanelli y Paradiso c. Italia.

${ }^{2}$ V. LoRubBio, "L'interesse superiore del minore come overruling "occulto"?. A proposito della Sentenza della Grande Camera (Corte E.D.U.). Paradiso e Campanelli c. Italia", Diritti Comparati

Working Papers, 2017; I. ANRò, "Surrogacy from the Luxembourg and Strasbourg perspectives: divergence, convergence and the chance for a future dialogue", Geneve Jean Monnet Working Papers, 09/2016, pp. 1-50; O. FERACI, "Maternità surrogata conclusa all'estero e Convenzione Europea di Diritti Dell'Uomo:Riflessioni a margine della Sentenza Paradiso e Campanelli contra Italia", $C D T$, vol. 7, n², 2015, pp. 420-439; C. HONORATI, "Paradiso e Campanelli c. Italia, atto secondo: la Corte EDU definisce la nozione di "vita familiare" e ribalta la sentenza precedente", Quaderni Constituzionali, nº 1, 2017, disponible en: http://www.forumcostituzionale.it/wordpress/wp-content/uploads/2001/01/honorati.pdf

${ }^{3}$ Casi todos los informes, resoluciones y sentencias reconocen el problema como transfronterizo, así por ejemplo, la resolución de la DGRN de 18 de febrero del 2019, lo pone de relieve desde sus inicios, "Instrucción de 18 de febrero de 2019, de la Dirección General de los Registros y el Notariado, sobre actualización del régimen registral de la filiación de los nacidos mediante gestación por sustitución", $B O E$ n $^{\circ} 45$, Sec. 1, 21 de febrero de 2019; L. BRUNET, "La globalization internationale de la gestation pour autrui", Travail, Genre et Societés, n², 2012, pp. 199-205; Y. ERGAS, "Babies Without Borders: Human Rights, Human Dignity, and the Regulation of International Commercial Surrogacy", Emory International Law Review, vol. 27, 2013, pp. 117-188; B. STARK, "Trasnational Surrogacy and International Human Rights Law", ILSA Journal of International and Comparative Law, vol. 18, 2012, pp. 369-386.

${ }^{4}$ Por ejemplo, párrafos 170 y siguientes de la STEDH.

${ }^{5}$ Párrafo 70 de la STEDH.
} 
incidental a las resoluciones de otros Estados donde se constituyó de forma legal la filiación mediante el contrato de gestación subrogada ${ }^{6}$. En realidad, el orden público internacional en estos supuestos, se representa en este principio supranacional del "interés superior del menor" como se expondrá en el último epígrafe, no pudiendo existir otros valores en estos casos que lo subordinen. Ahora bien, en CampanelliParadiso, se acusó al matrimonio de no haberla constituida de forma legal, por lo que, ¿qué sucede si la filiación de un menor nacido por esta técnica de gestación por sustitución no ha sido legalmente constituida en el Estado de origen?

3. Por otro lado, la valoración que hacen de forma particular, los jueces de la Gran Sala en qué debe considerarse como un "vínculo familiar" que, tendrá suma importancia, -según el TEDH-, para determinar finalmente si el menor nacido de la técnica de la gestación por sustitución puede o no considerarse hijo de los padres comitentes para darle un reconocimiento posterior en el Estado de destino, son también puestas de relieve por su relevancia y aportación ${ }^{7}$. Esto es, el TEDH lleva ya varias sentencias formando un concepto de familia "europeo" que emana de su jurisprudencia sobre el artículo 8 del CEDH (en relación a otros valores de tipo fundamental). La pregunta clave es entonces: ¿cuánto tiempo tiene que pasar para que un grupo de personas pueda ser considerado como "familia de facto"?

4. No obstante, la Gran Sala considera finalmente en el año 2017, que no existe por parte del Estado italiano, al menos, en este caso concreto, violación del artículo 8 del CEDH puesto que valora que no llegó a existir ningún vínculo familiar entre los padres comitentes y el menor, siendo este el aspecto más destacado del caso comentado ${ }^{8}$. Lo que nos hace volver a reflexionar con el mismo, unido a la jurisprudencia anterior, que debe existir un análisis del caso por el caso en estos supuestos y no aplicar soluciones de tipo general (one size fits all) como se concluirá en el presente análisis9.

5. También, para poder apreciar, hasta que punto el Estado de destino debe injerir o no en este "vínculo familiar", con la intención de tutelar el interés superior del menor, añadiéndose en cómo debe hacer dicha intervención en la vida privada y familiar cuando ya está previamente formada ${ }^{10}$. Esto es, cuando retira la tutela a los padres comitentes en base al respeto debido al interés superior del menor. Sobre este particular, habrá que recordar que no es la primera vez que el TEDH se pronuncia a favor del respeto de los derechos fundamentales subjetivos por encima de la actuación e injerencia que los Estados realizan mediante el mecanismo del orden público, como ya ponían de relieve, CALVo CARRASCoSA y CARRASCosa GoNZÁLEZ ${ }^{11}$.

${ }^{6}$ Vid. por todos, A-L. calvo caravaca y J. carrascosa González, "Gestación por sustitución y Derecho internacional privado. Más allá del Tribunal Supremo y del Tribunal Europeo de Derecho Humanos”, CDT, 2015, Vol. 7, №2, pp. 45-113; P. OREJUDO PRIETO DE LOS MOZOS, "Regognition in Spain of Parentage Created by Surrogate Motherhood", Yearbook of Private International Law, Vol. XII, 2010, pp. 619-637, esp. p. 635.

${ }^{7}$ I. BANTEKAS, "Children with dissabilities", I. BANTEKAs, M. AShley steIn y D. ANASTASIOU, The Convention on the Rights of Persons with Disabilities: A Commentary, 1a ed., Oxford University Press, 2018, pp. 198-229, esp. pp. 220-221; L. POLI, "La grande Camera e l'ultima parola sul caso Paradiso e Campanelli", SIDIBlog (Il Blog della Società italiana di Diritto Internationale e di Diritto dell'Unione Europea), Febrero 2017, disponible en: http://www.sidiblog.org/2017/02/21/la-grande-camera-elultima-parola-sul-caso-paradiso-e-campanelli__ : "L'elemento centrale del ragionamento compiuto allora dalla maggioranza dei guidice era rappresentato dall estensione del concetto di vita familiare alla situazione intercorrente tra i coniugi Campanelli ed il bambino, pur in asseza di consaguineità e formalizzazione giuridica del rapporto (par. 69). (...)" (el resaltado es de la autora); M. GERVASI, "The European Court of Human Rights Shaping Family Life in Cross Border Surrogacy: The Paradiso et Campanelli case", en E. Carpanelli y N. LazZerini (EDS.), Use and Misuse of New Technologies: Contemporary Challenges in International and European Law, Springer, 2019, pp. 151-166, esp. p. 155.

${ }^{8}$ G. PUPPINK, "Surrogacy: general interest can prevail upon the desire to become parents- about the Paradiso and Campanelli v. Italy Grand Chamber judgment of 24th January 2017”, Revue Lamy de Droit Civil, nº146, 2017, pp. 1-8.

${ }^{9}$ E. FARNós Amorós, "Paradiso y Campanelli c. Italia (II) : los casos difíciles crean mal derecho", Rev Bio y Der, n 40, 2017, pp. 231-242.

${ }^{10}$ Esto es, que medidas de protección que adopta para tutelar de un lado, el interés superior del menor y sus derechos fundamentales como el derecho a la vida privada y familiar reconocidos en textos de corte convencional y por ende, supranacionales. De otro lado, la tutela del orden público internacional en cuanto al reconocimiento que cabe o no de la filiación creada por maternidad surrogada en otro Estado donde esté permitida dicha técnica, cuando su ordenamiento jurídico lo prohíba y sean sus nacionales quien decidan acudir a estos Estados para obtener dicha filiación sobre estos menores.

${ }^{11}$ A-L. calvo caravaca y J. Carrascosa GonZÁLeZ, "Gestación por sustitución y Derecho internacional privado. Más allá del 


\section{Referencias sobre el supuesto de hecho}

6. Hechos del caso. Los señores Giovanni Campanelli y Donatina Paradiso, matrimonio italiano y residentes en Italia, decidió como muchas otras parejas, acudir a la técnica de la gestación por sustitución en Rusia en el año 2010, para tener un hijo, tras varios intentos infructuosos desde el año 2006 de tener hijos mediante la técnica in vitro e incluso, de haber esperado, un proceso de adopción en Italia. De hecho, debe ponerse de relieve que se les consideró aptos para adoptar (has been assessed fit to adopt), aunque no pudieron realizar ninguna adopción en Italia ${ }^{12}$.

Estando ya en Rusia, contactaron con una clínica especializada en la técnica de gestación por sustitución, consiguiendo mediante la misma, una donante del vientre materno a la que pagan entre 47.00050.000 euros. Esta madre gestante, además consta que renuncia a todo derecho de filiación sobre el menor el mismo día de su nacimiento de forma escrita en un documento privado (27 de febrero 2011$)^{13}$.

7. El problema o si se prefiere, cómo empezó el iter de este caso llegando finalmente al TEDH, devino cuando el progenitor comitente paterno intentó inscribir al menor en el Registro Civil italiano como su hijo en agosto del 2011, cuando ya se habían trasladado a Italia con el mismo, cuando éste tenía seis meses de edad. Cuando el señor Campanelli fue a registrar al menor en el registro correspondiente, el Encargado del Registro italiano, no sólo denegó dicha inscripción sino que acusó de fraude de ley, entre otros ilícitos ${ }^{14}$, a los señores Campanelli y Paradiso, dado que en el documento ruso que constituía la filiación entre los comitentes y el menor, no se había hecho constar que dicha filiación había sido realizada por la técnica de la gestación por sustitución. Al intentar justificar que el menor era su hijo tras las desaveniencias ocurridas en el Registro, se someten a un test de ADN, pero el mismo test mostró que no existía material genético de ninguno de ambos progenitores para sorpresa también del matrimonio que pensó que al menos, el menor tenía carga genética del progenitor paterno (el señor Campanelli) ${ }^{15}$.

8. Inicio del procedimiento ante los tribunales italianos. En noviembre del año 2011, el Tribunal de Menores de Campobasso (Italia) decide retirar de inmediato la custodia del menor al matrimonio y poner al menor en manos de los Servicios Sociales al mismo en una Casa Famiglia, sin contacto ninguno con el matrimonio Campanelli-Paradiso. El menor quedó sin ninguna identidad formal hasta el año 2013, en contra de lo establecido en el artículo 7 apartado $1^{\circ}$ de la Convención de los Derechos del $\mathrm{Niño}^{16}$, algo que las autoridades italianas consideraron responsables a los señores Campanelli-Paradiso por haber cometido fraude de ley. No obstante, pocos meses después de la retirada del menor a los comitentes, fue dado en acogida y posteriormente en adopción, a una nueva familia, con la que permanece desde su entrega. Entendiendo entonces que los nuevos vínculos familiares constituidos y la nueva identidad otorgada al menor se habían consolidado de forma durarera con la nueva familia, que el tiempo que había pasado bajo el cuidado y tutela de los señores Campanelli-Paradiso.

Tribunal Supremo y del Tribunal Europeo de Derechos Humanos”, CDT, 2015, Vol. 7, №2, pp. 45-11

${ }^{12}$ Este aspecto se tuvo en cuenta por el TEDH en su primera resolución del caso para valorar que la medida que adoptó el gobierno italiano de dar en adopción al menor, pero no permitir a los comitentes adoptarlo porque iba en contra del orden público internacional, en cuanto se les había acusado de fraude de ley; en Italia se ha tipificado como delito con seis meses de cárcel y multas pecuniarias, C. CAMPIGLIO, "Lo stato di figlio nato da contratto internazionale di maternità", Rivista di Diritto internazionale privato e processuale, 2009, pp. 589-604.

${ }^{13}$ FH 14 STEDH; P. Beaumont y K. TRIMmings, "The European Court of Human Rights in Paradiso and Campanelli v. Italy and the way forward for regulating cross-border surrogacy", CPIL Working Paper, no. 3, 2017, pp. 1-21.

${ }^{14}$ M. W. wINKLER, "Senza identità: il caso Paradiso e Campanelli c. Italia”, GenIUS Rivista di Studi Giuridici sull'orientamento sessuale e l'identità di genere, Vol. 1, 2015, pp. 243-258.

${ }^{15}$ Vid. infra, Epígrafe IV, APARTAdo 1; FH No14 STEDH as. Campanelli y Paradiso (2017); J. monteroni, "Un contrato internacional de maternidad subrogada. La decisión de la Gran Sala del Tribunal Europeo de Derechos Humanos en "Paradiso y Campanelli”, Prudentia Iuris, nº3, 2017, pp. 347-352.

${ }^{16}$ El artículo 7 de la Convención de los Derechos del Niño reza así: "1. El niño será inscrito inmediatamente después de su nacimiento después y tendrá derecho desde que nace a un nombre, a adquirir una nacionalidad y, en la medida de lo posible, a conocer a sus padres y ser cuidado por ellos. 2. Los Estados Partes velarán por la aplicación de estos derechos de conformidad con su legislación nacional y las obligaciones que hayan contraído en virtud de estos instrumentos internacionales pertinentes en esta esfera, sobre todo cuando el niño resultara de otro modo apátrida". 
9. La apelación. Los señores Campanelli y Paradiso apelaron tras el primer procedimiento, al Tribunal de apelación de Campobasso en el año 2012, pero el fallo siguió siendo el mismo de parte de estas instancias superiores. Ya desde los inicios, las autoridades italianas observaron este supuesto de hecho, más que un caso de gestación por sustitución un supuesto de adopción internacional, algo destacable por las manifestaciones de ambos tribunales ${ }^{17}$. En el año 2013, el Tribunal de menores de la misma localidad declaró que el matrimonio Campanelli-Paradiso, no tenía capacidad para adoptar a dicho menor puesto que nunca habían sido padres del mismo y tampoco se habían constituido como una familia (en contra de lo declarado en el año 2006 y que el TEDH observó ${ }^{18}$ ).

10. Inicio del procedimiento ante el TEDH. Es, a la luz de estas resoluciones italianas en contra de los pedimentos del matrimonio, más la reiterada negativa de las autoridades italianas de considerar su posición como padres comitentes, ni siquiera de poder optar por la posibilidad de llegar a ser adoptantes del menor que habían traído de Rusia, cuando el matrimonio decide interponer ante el TEDH un recurso en el que basa su defensa, que el gobierno italiano había y estaba violando los derechos fundamentales de los artículos 6, 8 y 14 del CEDH con su actuación.

11. En relación a estas peticiones realizadas por el matrimonio Campanelli-Paradiso, dos de las mismas no fueron admitidas a trámite por el TEDH, en el año 2015 y mantenidas en el año $2017^{19}$. La revisión del rechazo de las autoridades italianas de registrar el nacimiento del menor y el intento de los mismos de las acciones de filiación sobre el menor como una violación del artículo 6, por dos razones:

- Primera. El matrimonio no había agotado la vía de los recursos internos en su país de origen (en este caso, que no habían planteado ante el Tribunal de casación italiano ningún recurso), siendo este aspecto necesario para poder solicitar la tutela del TEDH ${ }^{20}$.

- Segunda. Al no constar que tenían vínculos biológicos con el menor, ni ninguna autorización formal como representantes del menor, no tenían capacidad para representarlo (esto es lo que considera el TEDH para rechazar estas peticiones).

12. Sin embargo, en el año 2015 , sí fue admitida a trámite la petición en relación a la violación del artículo 8 del CEDH apartado $1^{\circ}$, por considerar el TEDH que los comitentes y el menor si habían formado una familia de facto y que además, según se hace constar en los Fundamentos de Hecho y de Derecho, los comitentes habían formado un vínculo emocional con el menor mediante informe psicológico, así como eran importantes para el fondo del asunto ${ }^{21}$.

${ }^{17}$ P. Beaumont y K. trimmings, "The European Court of Human Rights in Paradiso and Campanelli v. Italy and the way forward for regulating cross-border surrogacy", CPIL Working Paper, no. 3, 2017, pp. 1-21; S. BASSAN, "Cross-border surrogacy transaction (CBST). Can consumers'states choose whether or not to regulate?", Völkerrechtsblog, julio 2016, disponible en: https://voelkerrechtsblog.org/cross-border-surrogacy-transactions-cbst/;_en respuesta a BASSAN, M. COTTIER, "Understanding the impact of different concepts of surrogate mother for the regulation of international surrogacy arrangments", Voelkerrechtsblog, 2016, disponible en: https://voelkerrechtsblog.org/understanding-the-impact-of-different-concepts-of-surrogate-mother-for-theregulation-of-international-surrogacy-arrangements/.

${ }^{18}$ I. ANRÒ, "Surrogacy from the Luxembourg and Strasbourg perspectives: divergence, convergence and the chance for a future dialogue", Geneve Jean Monnet Working Papers, 09/2016, p. 20, que pone de relieve que el TEDH no consideró que se les hizo aptos para la adopción, pero para adoptar menores a partir de cierta edad (en base a la limitación legal en la normativa italiana, para adoptar menores de un año de edad por personas que superen los 40 años de edad).

${ }^{19}$ FD 82 y SGS., STEDH as. Campanelli y Paradiso (2017), a pesar que las partes hicieron observaciones contra las peticiones rechazadas por la Sección Segunda del TEDH. El Gobierno italiano observa que las partes no agotaron los recursos internos en lo relativo al no reconocimiento del certificado de nacimiento ruso. Los señores Campanelli y Paradiso no habían apelado al Tribunal de Casación italiano, la decisión del Tribunal de Apelación de Campobasso de no reconocer el certificado de nacionamiento, como se informa en el texto.

${ }^{20}$ No obstante, la decisión del Tribunal de Apelación de Campobasso, no podía ser recurrida ante el Tribunal de Casación italiano, porque era provisional y no firme tal como se establece en el sistema procesal italiano; P. BEAUMONT Y K. TRIMMINGS, "The European Court of Human Rights...", loc.cit. supra ..., p. 3; I. ANRò, "Surrogacy from the Luxembourg and Strasbourg perspectives: divergence, convergence and the chance for a future dialogue”, Geneve Jean Monnet Working Papers, 09/2016, p. 19.

${ }^{21}$ FH 34 et seq., as. Campanelli y Paradiso (2017). 
13. A pesar que el TEDH reconoció que la retirada del menor a los comitentes se hizo de conformidad con la ley y que estos no tenían capacidad reconocida para representarlo ${ }^{22}$, si consideró que el Gobierno italiano no había tenido en cuenta ni los derechos ni el interés superior del menor sin que por ello, conllevase, según el TEDH, ninguna obligación para el gobierno italiano de devolver el menor a los comitentes, dado que había ya desarrollado vínculos familiares con la familia de acogida ${ }^{23}$. De hecho, también rechazó las observaciones del Gobierno italiano en contra de la demanda del matrimonio Campanelli-Paradiso.

\section{Las aportaciones del Tribunal Europeo de Derechos Humanos con el caso al debate de la ma- ternidad subrogada: el porqué del giro de su jurisprudencia}

\section{El Derecho al respeto a la vida privada y el derecho a la vida familiar: el Artículo 8 del CEDH}

14. El TEDH, en este caso, hace de nuevo un recorrido prolijo sobre el ámbito material del artículo 8 del CEDH, que ya desdibujó en su jurisprudencia anterior relativa a supuestos de gestación por sustitución ${ }^{24}$, en coordinación con el ámbito material de otras normas de corte convencional. En concreto, del artículo 3 de la Convención de los Derechos del Niño ${ }^{25}$.

Recuerda que el CEDH no establece una protección específica a los derechos de los niños, pero sí remite de forma tácita a la Convención de los Derechos del Niño que debe complementar en lo que respecta a la tutela de sus derechos fundamentales. En especial, su derecho a la vida privada y la vida familiar cuando ya está sólidamente formada ${ }^{26}$.

15. La Sentencia del año 2015. En el año 2015, la Sección $2^{\mathrm{a}}$ del TEDH, falló a favor de los padres comitentes, condenando al Gobierno italiano por haber violado el artículo 8 del CEDH y no haber

${ }^{22}$ FD 87 et seq. as. Campanelli y Paradiso (2017) pone de relieve que el interés superior del menor debe ser puesto de relieve ante la Gran Cámara, aunque estos no ostentasen de locus standi (capacidad de representación) según el Gobierno italiano en sus observaciones realizadas ante el TEDH: on the grounds that the applicants did not have locus standi before the Court.

${ }^{23}$ Parr 206, 210 y 213 as. Campanelli y Paradiso (2017).

${ }^{24}$ Cómo bien se conoce, todos los supuestos de gestación por sustitución que el TEDH ha analizado y ha resuelto han sido consecuencia de la denuncia de los padres comitentes en relación a una violación por parte de sus Estados del artículo 8 CEDH. Por citar algunos de los casos más importantes y renombrados así como más estrechamente relacionados: STEDH de 26 de junio 2014, as. Labasse vs. Francia; STEDH de 26 de junio 2014, as. Mennesson vs. Francia (FH 81 as. Campanelli y Paradiso (2017)-Comparative Law Material); Opinión de los jueces Gaetano, PINTO DE ALBURQuerque, woJTYCZeK y dedov (p. 54 as. Paradiso y Campanelli, 2017): "the application of Article 8 requires a careful definition of that provision's scope of application".

${ }^{25}$ En la STS de 6 de febrero de 2015, FD 6, se establece esta relación así como en toda la STEDH del caso analizado. La Convención de los Derechos del Niño (en particular, su artículo 7.1, que establece la obligación de inscripción de los niños, así como su derecho al nombre, nacionalidad, conocimiento de sus progenitores y el cuidado que estos deben guardarle, siendo obligación de los Estados establecer estos derechos del niño en sus legislaciones a la luz de las diferentes Convenciones internacionales que hayan podido firmar) se relaciona de forma efectiva con el artículo 8 de la CEDH, que supone obligaciones de tipo positivo para los Estados parte de la misma; A-L.CALVo CARAVACA y J. CARRASCOSA GOnŹ́lez, Derecho Internacional Privado, $16^{\mathrm{a}}$ ed., Vol. II, 2016, pp. 398-402; del artículo 3 se desprende que ofrece tres criterios (CALVO CARAVACA Y CARRASCOSA GONZÁLEZ, loc.cit., p. 84): 1. Criterio gestativo; 2. Criterio genético; 3. Criterio social-intencional. Siendo este criterio, el que interesaba en este supuesto dado que no existía ningún aporte genético por parte de ninguno de los comitentes); C. KALIN, "Transnational Surrogacy in the Light of the Case-law of the European Court of Human Rights", Journal of Siberian Federal University, Humanities \& Social Sciences, nº6, 2017, pp. 906-912, p. 909; I. ANRò, "Surrogacy from the Luxembourg and Strasbourg perspectives: divergence, convergence and the chance for a future dialogue", Geneve Jean Monnet Working Papers, 09/2016, pp. 17-18: "In the context of ECHR, the best interests of the child has been inserted through the case law of the EctHR in the interests that may justify a restriction to the family and private life of the individuals according to ECHR art. 8, despite the lack of reference in this article (...)"; en relación a los criterios de filiación, C. CAMPIGLIO, "Identitá culturale, diritti umani e diritto internazionale privato", Rivista de Diritto internazionale, Vol. 94, nº, 2011, pp. 1029-1064; id. "Norme italiane sulla procreazione assistita e parametri internazionali: il ruolo creativo dell giurisprudenza (Italian provisions on Assisted Procreation and International Parameters: The Creative Role of Courts)", Rivista de Diritto internazionale privato e processuale, 2014, pp. 481-516; "La filiazione nel diritto internazionale...", en Trattato de diritto de famiglia ... ob.cit.

${ }^{26}$ A. Young, "Comment, Advances in Children's Rights over the past decade: the Interamerican Court of Human Rights and the European Court of Human Rights'Progressive incorporation of the Convention on the Rights Children", Journal of the American Academy of Matrimonial Lawyers, vol. 28, 2015, pp. 285-307, p. 300 y p. 303. 
tenido en cuenta, con su actuación, el interés superior del menor. Esto es así, porque los jueces de la Sección $2^{\mathrm{a}}$ tras análisis del caso, consideraron que los ocho meses que habían pasado los padres comitentes con el menor eran suficientes para entender que se había constituido una familia de facto (en relación a la actuación de la autoridad italiana en quitarles al menor e impedir el desarrollo de una "vida familiar"27).

Un vínculo que consideraron, en esta primera resolución, que debía haberse respetado puesto que no había existido para el menor ningún riesgo grave y no se le había puesto en peligro por los progenitores comitentes.

16. El fallo del año 2017. Ahora bien, en el año 2017, la Gran Sala de dicho tribunal, ha dado fin a esta controversia considerando lo contrario, siendo mayoría los jueces que han votado en contra de lo que estableció la Sección $2^{\text {a28 }}$. Esto es, que no ha existido violación del artículo $8 \mathrm{CEDH}$ alejándose de la jurisprudencia anterior en supuestos no sólo de gestación por sustitución, pero, ¿por qué? ${ }^{29}$.

La mayor parte de los jueces de la Gran Sala considera en 2017 que no existía violación del artículo 8 porque el vínculo familiar no se había formado previamente como condición sine qua non para invocar la tutela del apartado $2^{\circ}$ del artículo 8 . El vínculo que se había formado era débil (sólo 5 jueces se mantuvieron disidentes sobre este particular de los 17 de la Gran Sala) y había sido considerado ilegal por las autoridades italianas que no le otorgaron como consecuencia, dicho reconocimiento ni validez necesarios para que produjese los mismos efectos en su territorio.

\footnotetext{
${ }^{27}$ Sección $2^{\mathrm{a}}$ del TEDH: "reiterated that the removal of a child from the family setting was an extreme measure, which could be justified only in the event of inmediate danger to the child"; no obstante, el procedimiento que duró dos años (antes de obtener esta primera sentencia), no condenaba al Estado italiano a devolver al menor a los comitentes, porque ya había sido entregado en adopción a otra familia, y posiblemente en dos años ya había formado vínculos familiares con esta nueva familia. Lo que pretendía el TEDH era generar estos derechos para futuras familias en casos similares, A. Young, loc.cit. supra, p. 303.

${ }^{28}$ Noticias que se han hecho eco de estos pronunciamientos y de la contradicción entre los mismos, antes del pronunciamiento del 2017, de la Gran Sala: Diritto Penale Contemporaneo, "La Corte Condanna 1'Italia in un caso di Maternità Surrogata all'estero", 3/02/2015, disponible en: https://www.penalecontemporaneo.it/d/3646-la-corte-edu-condanna-l-italia-in-uncaso-di-maternita-surrogata-all-estero : G. PUPPINCK, "La CEDH valide la vente d'enfant", La Village de la Justice, 4/02/2015; A. viviani, "Il caso Paradiso e Campanelli ovvero la Corte Europea contro i "pregiudizi” del Giudici Nazionali", SIDIBlog, 3/02/2015, disponible en: http://www.sidiblog.org/2015/02/03/il-caso-paradiso-e-campanelli-ovvero-la-corte-europea-controi-pregiudizi-dei-giudici-nazionali// S. FLORESCU, "What About the Children? Critical perspectives in the light of a new European judgment on surrogacy”, 28/04/2015, disponible en: https://leidenlawblog.nl/articles/what-about-the-children; R. vAUGHN, "ECHR Condemns Italy for Removing Child Born Via Surrogacy Abroad", International Fertility Law Group, 25/02/2015, disponible en: https://www.iflg.net/echr-condemns-italy-for-removing-child-born-via-surrogacy-abroad/; después del pronunciamiento de la Gran Sala de 2017: IDIBE. Noticias Legales, "El TEDH pone fin al conocido caso Paradiso vs. Italia, considerando que la decisión italiana de poner a cargo de los servicios sociales para dar en adopción a un niño nacido en Rusia mediante vientre de alquiler no vulnera el art. 8 de la Convención Europea de Derechos Humanos", 7/02/2017; R. ENGLISH, "Strasbourg grapples with international surrogacy and the concept of "family", UK Human Rights Blog, 21/05/2017, disponible en: https://ukhumanrightsblog.com/2017/05/21/strasbourg-grapples-with-international-surrogacy-and-the-concept-of-family/; D. ROMERO RIVERo, "La Corte Europea de Derechos Humanos y su nueva lectura de la gestación subrogada", Nexos, 21/02/2017, disponible en: https://eljuegodelacorte.nexos.com.mx/?p=6382 ; E.A. HEALTH, "European Court of Human Rights Rules Removal of Child Born via Surrogacy from Intended Parents Not a Rights Violation", ASIL, 10/02/2017, disponible en: https:// www.asil.org/blogs/european-court-human-rights-rules-removal-child-born-surrogacy-intended-parents-not-rights ; Global Health and Human Rights Database, "Paradiso and Campanelli v. Italy (ECHR 034 2017), disponible en: https://www.globalhealthrights.org/health-topics/health-care-and-health-services/paradiso-and-campanelli-v-italy/; de forma sucinta, G. NOTO LA DIEGA, "Same-sex adoptions in private international law: Good news from Italy", Inherently Human, 10/12/2017, disponible en: https://inherentlyhuman.wordpress.com/author/inherentlyhuman/page/1/.

${ }^{29}$ Algunos autores opinan, aunque con cautela, que se debió a no recomendar la gestación por sustitución como un recurso para tener hijos, esto es, por las cuestiones de corte ético, g. puppinck, "Surrogacy: general interest can prevail upon the desire....", loc.cit., p. 8: "In this case, the Choice of the Court was radically different, probably because the majority is no longer convinced that surrogacy would be advisable, even in the name of moral and economic liberalism"; D.DE CARLUCCI, A. HERRERA, M. LAMM Y E. DE LA TORRE, "La gestación por sustitución en el Tribunal Europeo de Derechos Humanos. A propósito del caso "Paradiso y Campanelli c. Italia", Diario La Ley, 2017; E. LAMm y N. RUBAJA, "BIOÉTICA EN LOS TRIBUNALES. Parámetros jurisprudenciales en los casos de gestación por sustitución internacional. Los lineamientos del Tribunal Europeo de Derechos Humanos y sus repercusiones en el Derecho global", Rev. Bio y Der.,vol. 37, 2016, pp. 149-170; CEDH, "L'éloignement par les authorités d'un enfant né de GPA sans lien biologique avec les parents d'intention n'a pas été contraire à la Convention européenne des droits de l'homme", 034 (2017).
} 
17. Por lo tanto, al no encontrar indicio de vida familiar válidamente constituida no tenía porque otorgarse dicha protección en contra de la actuación del gobierno italiano ${ }^{30}$. Esto es, que el gobierno italiano, en este caso concreto, tenía derecho a retirar la custodia del menor al matrimonio, añadiéndose además que no existía vínculo genético de ningún comitente en el menor. La actuación de las autoridades italianas se basaba en proteger su derecho a la identidad (art. $7 \mathrm{CDN}$ ), que los padres comitentes habían puesto en peligro con sus actuaciones fraudulentas.

Para el TEDH el mero hecho que los comitentes estuvieran desde el inicio en Rusia, no era una razón de peso para entender que se había formado dicho vínculo familiar por las cuestiones relativas al fraude de ley cometido por el matrimonio.

18. Por otra parte, el derecho a la vida privada en cuanto a que la injerencia de los Estados en la vida privada y familiar entre un grupo de personas, debe estar siempre justificada por razones que se sitúan por encima de los intereses privados o de los particulares. En estos casos, dice el TEDH que existen dos. Los de los menores, y los de los padres comitentes ${ }^{31}$. Y, siempre tendrán preferencia por la aplicación debida del principio del interés superior del menor, los intereses de los menores.

\section{Determinación de la duración del "vínculo familiar" y el concepto de familia de facto en el De- recho europeo}

\section{A) Qué debe entenderse como una familia de facto según el artículo 8 del CEDH}

19. Al hilo de lo establecido en el anterior apartado ${ }^{32}$, es aquí donde se encuentra la vexata quaestio del caso, en lo que a la aparente contradicción de las dos resoluciones del TEDH sobre que debe entenderse como "vinculo familiar" tanto en el Estado de origen como en el Estado de destino donde, en principio, la gestación por sustitución no se permite o no se regula. De hecho, es el vínculo familiar de facto el que valora el TEDH en estos supuestos así como de vínculos constantes y cercanos entre dicho grupo de personas, aunque en este supuesto lo hace con cautela ${ }^{33}$.

20. El artículo 8 del CEDH, -explica el TEDH-, no viene a tutelar el "derecho/deseo a ser padres" ${ }^{34}$, sino el derecho que cuando una familia se ha constituido de forma válida sea respetada por los Estados, incluyendo con ello la vida privada que se establece con el desarrollo del vínculo familiar. Siendo este aspecto enfocado en estos supuestos de gestación por sustitución especialmente, a tutelar los

\footnotetext{
${ }^{30}$ Por ejemplo, D. Romero RIVERo, "La Corte Europea de Derechos Humanos y su nueva lectura de la gestación subrogada...", cit. supra: "Si es suficiente para crear, ilegalmente, un vínculo con el niño en el extranjero para que las autoridades nacionales se encuentren obligadas a reconocer la existencia de "vida familiar", entonces está claro que la libertad de los Estados de no dar efectos jurídicos a la gestación por sustitución, no obstante que ha sido reconocida por la Corte en sus precedentes (vid. Mennesson v. France (...), y Labasse vs. France (...), es reducida a la nada".

${ }^{31}$ Parr 205 as. Campanelli y Paradiso (2017).

${ }^{32}$ Véase por ejemplo su jurisprudencia anterior desde el caso Menesson que la Corte sí considera que existió vínculo familiar suficiente para que el Estado no pudiese sustraer al menor de sus padres comitentes, porque ello iría en contra del "interés superior del niño"; un comentario del affaire Menesson desde el prisma español, J. FLORES RODRíGUEZ, "Vientres de alquiler: más cerca de su reconocimiento legal en Europa. Comentario a la STEDH de 26 de junio de 2014, recurso no 65192/11", Diario La Ley, Sección Tribuna, 2014. También como en Italia y España, en Francia recaer la prohibición de orden público sobre este tipo de obtener la filiación pero en el mismo Código civil francés en su artículo 16-9).

${ }^{33}$ Opinión de los jueces Gaetano, PINTO DE AlbuRQuerque, WOJTYCZEK y DEDOv, p. 52: "2. According to the jugdement, the existence or non-existence of family life is esentially a question of fact, depending upon the existence of close and constant personal ties" (remitiendo al párrafo 140 de la Sentencia); Pero no tiene en cuenta, -según ANRò-, si puede existir tráfico de menores o el padre comitente haya sido juzgado en su país de origen por haber cometido ciertos ilícitos contra menores o que no existiese ningún vínculo genético, cuestión que también puede afectar al derecho de los niños a conocer su identidad según la Convención de los Derechos del Niño, I. ANRò, "Surrogacy from the Luxembourg and Strasbourg perspectives: divergence, convergence and the chance for a future dialogue", Geneve Jean Monnet Working Papers, 09/2016, p.33.

${ }^{34}$ La Gran Sala aclara con precisión este aspecto: "Las disposiciones del artículo 8 no garantizan ni el derecho a fundar una familia ni el derecho a adoptar (...) El derecho al respeto de la vida familiar no protege el simple deseo de fundar una familiar: este presupone la existencia de una familia (...), o por lo menos la relación potencial que se pudiera desarrollar, por ejemplo, entre un padre natural y un hijo nacido fuera de matrimonio (...)"; parr. 140 et seq as. Campanelli y Paradiso (2017).
} 
derechos del menor en un Estado de destino, donde acabará desarrollando este vínculo familiar con sus padres comitentes y donde puede no llegar a ser reconocido como hijo de los comitentes.

21. Los Estados que prohíben la técnica, lo que en realidad prohíben es la renuncia de la filiación por parte de la madre gestante, como se conoce. Entonces, si la madre gestante ha renunciado a su derecho mediante contrato de forma legal en el Estado de origen, el riesgo se encuentra en que puede dejarse al menor en riesgo de no tener una identidad formal en contra de la Convención de los Derechos del Niño, por no reconocer tampoco la filiación de la madre comitente. Al menos, hasta que no se de validez y efectos a dicha resolución en el Estado de destino, la situación en la que pueden quedar los menores es una situación contraria a lo establecido en los textos convencionales.

22. Este aspecto es fundamental para que pueda entenderse que, entre las partes, -padres comitentes y menor nacido de esta técnica-, formaban una verdadera familia en el Estado donde se constituyó la filiación (aunque sean nacionales de otro Estado).

23. De esta forma, también lo ponen de relieve los informes y el estudio que la "Conferencia de la Haya" ha venido realizando desde el año 2012 sobre la gestación por sustitución, especialmente los últimos, realizados en el año $2014^{35}$ y el año 2015. Esto es, no se puede discriminar a un grupo de personas que se han constituido como familia atendiendo a la formación del vínculo (matrimonio, adopción o por técnicas de fecundación in vitro y gestación por sustitución) puesto que esto iría en contra del artículo 14 del CEDH, siendo una discriminación ${ }^{36}$. Como se entiende también que es discriminatorio en contra del artículo 14 del mismo instrumento jurídico (prohibición de discriminación), entender que el artículo 8 del CEDH, tutela sólo la vida familiar que se ha constituido por vía del matrimonio celebrado de forma tradicional ${ }^{37}$.

\section{B) Qué no se entiende como una familia de facto según la jurisprudencia del artículo 8 del CEDH. Relación con el derecho a la vida privada}

24. Para el TEDH la duración del vínculo, como cuestión es muy importante para valorar si existió o no, vínculo familiar. De ahí que finalmente se pueda determinar en qué casos hay violación del artículo 8, y en qué supuestos no existe dicha violación y la actuación del Estado es correcta. No obstante, se muestra reticente a considerar que tiene que haber un tiempo predeterminado por la ley ${ }^{38}$. Sobre esta cuestión en particular, se plantea también de forma sucinta, hasta que punto el Estado debe injerir en la vida privada y familiar, algo que pertenece a la esfera más íntima de los sujetos.

25. Además, la valoración del vínculo familiar no debe hacerse de conformidad al vínculo emocional sino a la duración y la calidad del mismo. La calidad se sopesa, dice el TEDH, en relación al cuidado debido que los padres o tutores legales han dado al menor. No puede valorarse únicamente en rela-

${ }^{35} \mathrm{HCCH}$, "The Parentage/Surrogacy Project:an Updating Note (Le Project Filiation/Maternité de Substitution: Note de mise à jour)", Prel. Doc. No 3A (Doc. Prél. Doc. No 3A), Febrero 2015, pp. 1-17, disponible en: https://assets.;.net/docs/82d31f31294f-47fe-9166-4d9315031737.pdf; S. BASSAN, "Cross-border surrogacy transaction (CBST). Can consumers'states choose whether or not to regulate?”, Völkerrechtsblog, julio 2016, disponible en: https://voelkerrechtsblog.org/cross-border-surrogacy-transactions-cbst/ ; los últimos proyectos de la Conferencia de la Haya en maternidad subrogada están disponibles en su página web y son relativamente muy recientes, de marzo de 2019: https://www.hcch.net/es/projects/legislative-projects/ parentage-surrogacy/surrogacy-2011-2015.

${ }^{36}$ A. Young, "Comment, Advances in Children's Rights over the past decade: the Interamerican Court of Human Rights and...", loc.cit., p. 304, al hilo del caso Pla and Purcernau vs. Andorra, en donde se discriminó en la herencia a un menor que no había nacido en un matrimonio válidamente celebrado bajo la forma canónica y otros casos más recientes relativos a la discriminación que habían estado sufriendo los hijos no nacidos en matrimonio (hijos extramatrimoniales), como el caso Fabris v. France en el año 2013.

${ }^{37}$ A.L. Calvo caravaca y J. carrascosa GonzÁlez, "Gestación por sustitución y Derecho internacional privado. Más allá del Tribunal Supremo y del Tribunal...”, loc.cit., p. 101 y siguiendo lo establecido en la primera resolución del caso en el año 2015.

${ }^{38}$ Parr. 153 as. Campanelli y Paradiso (2017): "It would admittedly appropiate to define a minimal duration of shared life which would be necessary to constitute de facto family, given that the assessment of any situation must take account of the "quality". " 
ción al vínculo emocional propiamente dicho que se crea entre un grupo de personas que conviven con el paso del tiempo, por no ser tutelado en el artículo 8 del CEDH el "deseo" o "intención de ser padre" 39.

26. Entonces, en este orden de ideas, lo que queda claro del pronunciamiento es que sólo en caso de existir peligro para el menor, un peligro grave real, es cuando esta injerencia estatal debe producirse para tutelar sus derechos de tipo fundamental (no more, no less). Derechos, que en principio corresponde velar a los progenitores o tutores, y que si no se hace así, entonces será el Estado el que debe tutelarlos, pero en casos de urgencia y necesidad siempre, según el apartado $2^{\circ}$ del artículo 8 del CEDH ${ }^{40}$.

27. En el caso Campanelli y Paradiso el hecho que tampoco existiese carga genética en el menor, como se presupone que debe existir en los casos de gestación por sustitución a la luz de las legislaciones de los Estados que sí la regulan y permiten para poder considerar que se ha creado un vínculo de filiación y con ello una "familia de facto", no fue sólo lo que se valoró para entender que el gobierno italiano había actuado e injerido en su vida privada y familiar de forma correcta. Tampoco el artículo 8 tutela familias de tipo tradicional. Tutela cualquier tipo de familia que tenga o no vínculos genéticos ${ }^{41}$.

En realidad, lo determinante para valorar que la separación entre este grupo de personas (padres comitentes y menor), no se constituía una medida del gobierno italiano desproporcionada para el interés superior del menor, fue la actuación del matrimonio Campanelli-Paradiso ${ }^{42}$.

Pero no así, en relación a su derecho fundamental de tener una vida privada. Se desprende de la sentencia del 2017, en cuanto explica que el menor, en este asunto, no se consideró como parte en el proceso (demandante) representado por sus padres comitentes, porque no fue reconocido como hijo legítimo, por lo que sólo se pudo tutelar en este caso, el derecho a tener una vida privada de los comitentes en su interacción con el menor. Diferenciando entonces dos derechos fundamentales diferentes pero relacionados en el mismo artículo en el mismo nivel de protección ${ }^{43}$.

\section{Incidencia del pronunciamiento en el Derecho doméstico de los Estados parte del Convenio Europeo de Derechos Humanos}

\section{El correctivo del orden público internacional (de protección) en el Estado de destino: la opinión del Tribunal Europeo de Derechos Humanos en el caso}

28. Se llega en este epígrafe a ciertas de las cuestiones más polémicas en materia de gestación por sustitución que tienen relación con el DIPr. Como ya se puso de relieve, se centran en el ámbito del reconocimiento y validez de resoluciones extranjeras. Teniendo en cuenta que la determinación del orden público internacional como concepto jurídico indeterminado y abstracto, es una operación complicada y debe ser precisada siempre a la luz del caso por caso $^{44}$.

\footnotetext{
${ }^{39}$ M. GERVASI, "The European Court of Human Rights Shaping Family Life in Cross Border Surrogacy...", en E. CARPANELLI Y N. LAZZERINI (EDS.), Use and ...., op.cit., esp. p. 155: “(...) in spite of the strenghts of the emotional bonds”.

${ }^{40}$ FH 63 STEHD as. Campanelli y Paradiso (2017).

${ }^{41}$ Parr 149 as. Campanelli y Paradiso (2017).

${ }^{42}$ Ibid (GERVASI) p. 157; párrafo 204. "In sum, for the domestic courts the primary concern was to put an end to an illegal situation. Having regard to the considerations set out above, the Court accepts that the laws which had been contravened by the applicants and the measures which were taken in response to their conduct served to protect very weightly public interests"; y, párrafo 215 (opinión final de la Gran Sala) en el as. Campanelli-Paradiso (2017).

${ }^{43}$ Parr 208: "Before entering into the question of whether the Italian authorities duly weighed the different interests invol$v e d$, the Court reiterates that the child is not an applicants'family within the meaning of Article 8 of the Convention."

${ }^{44}$ J. LAfFRANQUE, "A Look at the European Court of Human Rights Case Law on Moral Issues and Academic Freedom”, Juridica International, $\mathrm{n}^{\circ} 26,2017, \mathrm{pp} .34-46, \mathrm{p} .36$ : "This is why the third aspect of ethics in the Court is very vital: the ethics of decision making within the European Court of Human Rights - the issues of convintion and emotions on one hand and responsibility and rationality on the other, finding the right balance between generalisation and decising an individual case"; también puesto de relieve por la Resolución de la DGRN, 21 de febrero, cit. : “( ...) todo ello hace necesario un tratamiento que permita valorar todas las circunstancias que se presenten en cada supuesto, con una prueba válida y suficiente de los hechos, datos y declaraciones de voluntad que concurran en el mismo (...)"; V. LORUBBIO, "L'interesse superiore del minore come overruling "occulto"?. A propo-
} 
29. Interesa destacar también que el orden público internacional se ha modernizado. Ya no es un orden público internacional de dirección (y con cierta orientación material que fomenta el nacionalismo jurídico o legeforismo) sino de protección (que protege y tutela valores que no sólo se consideran estatales, sino supranacionales como el "interés superior del menor").

30. En este supuesto, el problema de la resolución en la que se hacía constar en Rusia, y bajo el Derecho ruso que los padres comitentes era el matrimonio Campanelli-Paradiso ${ }^{45}$, era que se había omitido que dicha filiación se había realizado mediante un contrato de gestación subrogada donde la madre biológica renunciaba a sus derechos de filiación sobre el menor. No obstante, según el Derecho ruso, la filiación estaba constituida de forma correcta. Por ello, no había dudas que los padres comitentes se habían constituido como tales, de forma legal ${ }^{46}$.

31. Entendiendo entonces el Encargado del Registro Civil en Italia que se había hecho en fraude de ley. En este sentido, cabe recordar el análisis que ya previamente realizaron CALVo CARAVACA y CARRASCOSA GONZÁLEZ en cuanto ${ }^{47}$, a que lo que hacen las Autoridades de los Registros no es enfrentarse a problemas de Derecho civil, sino de Derecho internacional privado, y en estos casos, modulado por el artículo 3 de la Convención de los Derechos del Niño. Añadiendo que, el interés superior del menor ya "viaja" en el contenido de la resolución extranjera, y que no puede ser anulado con argumentos relativos a la prevención del bad Forum Shopping o uso fraudulento por parte de los progenitores ${ }^{48}$, en cuanto no tiene relación con la filiación de los menores y con el orden público internacional en estos casos, que es el interés superior del menor, teniendo primacía sobre estos argumentos ${ }^{49}$.

32. Con ello, lo que se viene a decir es que, si los padres comitentes consiguieron la resolución y con esto, la filiación en Rusia, de forma legal, cumpliendo con los requisitos de dicho ordenamiento jurídico, el Encargado del Registro Civil, no debe centrarse en los aspectos sustantivos, sino formales o de reconocimiento en el caso que nos ocupa, evitando "judicializar la función registral", puesto que de lo que se trata es de valorar los efectos constitutivos de dicha certificación con el orden público del Estado de destino, porque ya se aplicó un Derecho al fondo del asunto para constituir dicha filiación.

sito della Sentenza della Grande Camera (Corte E.D.U.). Paradiso e Campanelli c. Italia”, Diritti Comparati Working Papers, 2017; D. GARCÍA SAN JOSÉ, "Epigenética y gestación por sustitución: más razones a favor de una regulación internacional para un negocio global", Anuario Mexicano de Derecho Internacional, vol. XII, 2017, pp. 329-368, que se centra en que el Convenio ad hoc de derechos en gestación subrogada debe proteger el justo equilibrio y tener en cuenta las cuestiones de tipo epigenético; J. FLORES RODRÍGUEZ, "Vientres de alquiler: más cerca de su reconocimiento legal en Europa. Comentario a la STEDH de 26 de junio de 2014, recurso no 65192/11", Diario La Ley, Sección Tribuna, 2014; I. ANRò, "Surrogacy from the Luxembourg and Strasbourg perspectives: divergence, convergence and the chance for a future dialogue", Geneve Jean Monnet Working Papers, 09/2016, pp. 29-33; L. POLI, "Maternità surrogata e diritti umani: una pratica controversa che necessita di una reglamentazione internazionale", Biolaw journal-Rivista de Bio Diritto, n³, 2015, pp. 7-28.

${ }^{45}$ Vid. FH n ${ }^{\circ} 8$ (Facts) STEDH as. Campanelli y Paradiso (2015).

${ }^{46}$ FH 73 STEDH as. Campanelli y Paradiso 2017; El Código civil ruso establece que los padres comitentes aparecerán como los padres del menor nacido de la gestación por sustitución, cuando la madre haya dado su consentimiento por escrito. No obstante, en el año 2011 nada establecía que los padres debían haber aportado material genético. Este aspecto cambia en el año 2012 en la Ley de Protección de la Salud de los ciudadanos en Rusia que introduce la disposición que la gestación por sustitución es aquella en la que una mujer gestante dona su vientre mediante contrato para hacer nacer el hijo de un matrimonio que ha ofrecido su material genético (traducción propia).

${ }^{47}$ Aún en relación a lo establecido en el ordenamiento jurídico español, puede ser extrapolable a lo sucedido en Italia, por ser este ordenamiento y el caso muy similar, A.-L. CALVO CARAVACA y J. CARRASCOSA GONZÁLEZ, "Gestación por sustitución y Derecho internacional privado. Más allá del Tribunal Supremo y del Tribunal Europeo de Derechos...”, loc.cit., pp. 63-66, esp. p. 65: "La instrucción aborda problemas de Derecho internacional privado, no de Derecho civil (...). No potencia ni permite el fraude a la ley española, sino que trata de dar solución a casos en los que la filiación de los nacidos en el extranjero tras una gestación por sustitución ha sido legalmente fijada", y p. 66, párr. 39: recordando la "tormenta del orden público internacional y español desatada"; J-F. RODRIGUEz, "Vientres de alquiler: más cerca de su reconocimiento legal en Europa. Comentario a la STEDH de 26 de junio de 2014, recurso nº 65192/11", Diario La Ley, Sección Tribuna, No8363, Año XXXV

${ }^{48}$ V.gr., sobre este particular, G. CUNIBERTI, "Note sous Cour d'Appel de Paris, 1er Chambre, du 25 oct. 2007", Clunet, 2008, pp. 145-153.

${ }^{49}$ Ibid. (CALVO/CARRASCOSA, p. 86, citando a J.M.tORRES PEREA: "puede considerarse que cuando una norma imperativa colisiona frontalmente con la cláusula del interés superior del menor puede quedar neutralizada su aplicación por exigirlo dicho principio que ha de considerarse prioritario”) y p. 91. 
33. Por ello, se valora que el problema que se presenta es un problema de reconocimiento de resoluciones, no de ley aplicable, dado que no se hace un análisis del Derecho aplicable al fondo del asunto y que debe ser matizado siempre para evitar entrar en un círculo sin salida, sin solucionar el verdadero problema ${ }^{50}$.

34. Atendiendo a lo anterior, se deben hacer dos reflexiones importantes, antes de aplicar el correctivo del orden público internacional sin tener en cuenta el principio del interés superior del menor, que en la primera resolución de la Sección Segunda del TEDH se puso de relieve también ${ }^{51}$.

- Primera: de la correcta aplicación de la técnica del reconocimiento y que presupuestos conlleva, entre ellos, la prohibición de revisión sobre el fondo del asunto.

- Segunda: valorar que ámbito territorial dispone la normativa del Estado de destino que prohíbe o no regula la gestación por sustitución. Esto es, si alcanzan a supuestos que sus nacionales realizan en el extranjero siendo por tanto normas que con alcance extraterritorial, o son normas con vocación territorial, como es en el caso español, el artículo 10 de la Ley de Reproducción Asistida ${ }^{52}$. Normas que sólo pueden aplicarse a supuestos que sólo se hayan realizado en el territorio español. La legislación italiana que prohíbe la constitución de filiación por medio de técnica de maternidad subrogada también es de alcance territorial limitado.

35. Entre la primera y segunda opción, la segunda opción es la se considera correcta, puesto que ambas normas (italiana y española) se han realizado sin vocación universal, aparte que no son normas de conflicto, que determinen la ley aplicable a los contratos de la gestación por sustitución. Tampoco se cuenta todavía con normas de conflicto específicas y las relativas a la filiación natural y por adopción en estos supuestos no tienen cabida (tampoco por analogía iuris), por sus diferencias, aunque ha sido una de las soluciones propuestas por algunos autores, aunque más por aquellos que consideran que el problema no es de reconocimiento y validez de resoluciones extranjeras sino de ley aplicable.

\section{Aportaciones de la Opinión particular de los Jueces del caso}

36. El TEDH pone de relieve con énfasis, que el "interés superior del menor" es un valor supranacional que los Estados parte del CEDH y de la Convención de los Derechos del Niño (de nuevo, en torno el artículo 3 apartado $1^{053}$ ) deben respetar siempre. Por ser estos textos convencionales, como se conoce, de preferencia de aplicación en el sistema de prelación de fuentes de los ordenamientos jurídicos de los Estados parte de los mismos ${ }^{54}$.

\footnotetext{
${ }^{50}$ No se trata, -como explican calvo Caravaca y CARRASCosa GonZÁlez-, en su análisis de la STS Sala de lo Civil de 6 de febrero de 2014, de entrar a determinar la filiación de nuevo, una filiación que ha sido ya determinada de conformidad con el ordenamiento jurídico del Estado de origen, sino de la validez extraterritorial de dicha determinación de la filiación que es legal en el Estado de origen. Ni siquiera, como estableció el Alto Tribunal, se aplican las normas de conflicto españolas (art. 9.4 CC) ni el Derecho sustantivo (art. 10 bis Ley 14/2006), por ello, no debería ser observado como un problema de ley aplicable; G. CUNIBERTI, "Note sous Cour d'Appel de Paris, 1er Chambre, du 25 oct. ....", loc.cit., p. 148.

${ }^{51}$ Párrafos 200 y siguientes, esp. p. 202: “(...) the Italian legislature was seeking to protect the best interests of the child as required by Article 3 of the Convention on the Rights of the Child"; parr 57 as. Campanelli y Paradiso (2017).

${ }^{52}$ Ley 14/2006, de 26 de mayo, sobre técnicas de reproducción asistida, BOE, nº126 de 27 de mayo de 2006; L. PERTUSA RODRÍGUEZ, "La dimensión consular de la gestación por sustitución en Derecho internacional privado", CDT, 2018, Vol. 10, №2, pp. 597-614; En Italia, Ley de 19 de febrero n40, "Norme in materia di procreazione medicalmente asistita", Gazzetta Ufficiale, $\mathrm{n}^{\circ} 45$, del 24 de febrero de 2004, disponible en: http://www.parlamento.it/parlam/leggi/040401.htm ; C. CAMPIGLIO, "VIII. La filiazione nel Diritto internazionale Privatto", en G. ColluRA, P. zatTI, L. Lenti y M. MANTOVANi (eds.), Trattato di Diritto de Famiglia. Filiazione (vol.2), Giuffré Editore, 2012, pp. 733-767, pp. 751-752.

${ }^{53}$ En el FH parr. 77, el TEDH reproduce además el Protocolo $n^{\circ} 7$ (2005) del Comité de los Derechos del niño de las Naciones Unidas en cómo debe ser implementado el art. 3 de la Convención de los Derechos del niño, por los Estados parte: “Article 3 sets out the principle that the best interests of the child are a primary consideration in all actions concerning children (...) The principle of best interests appears repeatedly within the Convention (including in articles 9. 18, 20 and 21) which are the most relevant to early childhood). The principle of best interests applies to all actions concerning children and requieres active measures to protect their rights and promote their survival, growth, and well being, as well as measures to support and assist parents and others who have day-to-day responsability for realizing children's interest"; y, en el parr. 208.

${ }^{54}$ En los siguientes Fundamentos de Hecho, el TEDH hace un repaso a la legislación convencional que deben los Estados
} 
No obstante, el equilibrio que el Estado debe tener entre el respeto del orden público internacional del Estado de destino y el interés superior del menor es fundamental ${ }^{55}$.

37. Según la opinión particular de los jueces, aunque nada impide que los Estados parte de la CEDH legislen como deseen la situación y tratamiento de los nacidos por la técnica de gestación por sustitución así como la determinación de la filiación (de hecho el TEDH no puede tampoco prohibir a los Estados partes, por su función), sí deben tener en cuenta antes de no dar reconocimiento ni validez a una resolución de otro Estado donde se constituyó dicha filiación y vida familiar a no respetar este derecho fundamental. Especialmente, en supuestos internacionales ${ }^{56}$.

\section{Conclusiones}

38. Primera.-Concepto de familia en el Derecho europeo: Una de las conclusiones que se extrae del análisis de la STEDH y que ya fue puesta de relieve por la doctrina y jurisprudencia es, que estos supuestos deben ser analizados de forma separada a la luz de las circunstancias de cada uno de ellos. Valorar si existe o no un "vínculo familiar" (familiar bonds) para distinguir si entre los padres comitentes y el menor se había formado una familia de facto no puede ser entendido por igual en todos los casos.

Por ello, limitarse a aplicar las normas a todos los supuestos de forma general, el orden público internacional y el interés superior del menor como un principio abstracto y general (one size fits all), conlleva al fracaso y a soluciones no equitativas en aquellos Estados donde la técnica o no está permitida o no está regulada. Campanelli y Paradiso es un claro ejemplo de este análisis del caso por el caso, en el que no se pudo considerar la verdadera existencia de un vínculo familiar por todos los datos aportados.

A su vez, parece que el TEDH, mediante la jurisprudencia de los supuestos relativos al ámbito de la filiación determinada por vínculos no genéticos como la adopción y los de gestación por sustitución, está construyendo un concepto de "vida familiar" a la luz del artículo 8 del texto convencional, que deberá tenerse en cuenta por los Estados parte a la hora de valorar el caso por caso en estos supuestos.

39. Segunda. Orden público internacional y fraude de ley: De nuevo, recuerda el TEDH que el recurso al orden público internacional para negar los efectos de este tipo de filiación constituida de forma legal en el Estado de origen, no se puede invocar por los Estados de destino como resorte automático para no otorgar reconocimiento a dicha filiación, porque vulnera los derechos del artículo 8 del CEDH de los menores y comitentes, y muy en relación al interés superior del menor como principio supranacional establecido en el art. $3 \mathrm{CDH}$. La CEDH protege los derechos fundamentales especialmente en casos internacionales, por lo que los Estados parte deben respetarlo. Tampoco deberían negar la validez de estas resoluciones, como medio disuasorio a sus nacionales de poder constituir este vínculo familiar en un Estado donde sí se permite. No obstante, en supuestos excepcionales como en el fraude de ley también influye en la relación formada entre los padres comitentes y el menor y ello si es motivo para no

parte de la CEDH y de dichos instrumentos respetar siempre y cuando sean parte, en los supuestos de gestación por sustitución, en relación a los menores, aunque en los mismos Estados la técnica esté prohibida por ley o no tolerada pero sin una prohibición expresa, así, en el FH 75 (Sobre la Convención de la Haya sobre abolición del requisito de legalización de documento público extranjero de 1961); FH 76 y 77 (Convención de los Derechos del niño, artículo 3, 7, 9, 20 y 21); FH 78 ( Convención de la Haya sobre protección de menores y adopción internacional de 1993) FH 79 (Principios por el Comité de expertos $A d$ Hoc del Consejo de Europa sobre el progreso de la Biomedicina); STEDH as. Campanelli y Paradiso (2017); A.-L.CALVo CARAVACA Y J. CARRASCOSA GONZÁLEZ, “Gestación por sustitución y Derecho internacional...”, loc.cit., esp.p. 66 y p. 82: “Pues bien, en el escenario del sistema jurídico español, el "interés superior del menor" constituye un "principio superior". Ello no significa que, en el caso concreto en el que se produzca un enfrentamiento entre estos dos principios antitéticos (= principio uno: el castigo de movimientos elusorios de la Ley española como el Forum Shopping y la correlativa protección de la "autoridad de la Ley española", y principio dos: el interés del menor), siempre prevalece el principio del interés del menor"; a. young, "Comment, Advances in Children's Rights over the past decade: the Interamerican Court of Human Rights and...", loc.cit.

${ }^{55}$ I. RIVERA, "Affaire Campanelli e Paradiso c. Italie: la Corte EDU torna a pronunciarsi sulla maternità surrogata e sul best interest of child come limite all'ordine pubblico internationale", federalism.it, $\mathrm{n}^{\circ} 3,2015, \mathrm{pp} .2-23$

${ }^{56}$ V.gr., en España, lo que se trata es decidir no es la filiación de los menores, ya constituida de forma legal en el país de origen, sino de aceptarla o rechazarla pero sobre la base de la técnica del reconocimiento de decisiones. 
valorar que se constituyó ninguna filiación entre ellos (aunque queda la de la madre gestante) ni ninguna familia de facto para invocar la tutela del artículo 8 del CEDH.

$\mathrm{Si}$ las autoridades consideran que este vínculo no fue válidamente formado ni siquiera en el Estado de origen, a la luz de los datos y documentos aportados, como en el supuesto de Campanelli y Paradiso, entonces, el Estado de destino, tiene que entrar a valorar que debe proteger al menor frente a una incertidumbre de poder dejarle sin identidad por un acto realizado por los padres comitentes del que no es responsable. Teniendo en cuenta que por encima del derecho a la vida privada y familiar, se tutela el interés superior de un menor contra actos que pueden perjudicarle gravemente.

40. Tercera.- Si bien es cierto que no era objeto de análisis otros aspectos relativos a los problemas que se suscitan en el ámbito del DIPr, la Doctrina especializada en esta materia, propone de lege ferenda una ley o norma que regule los aspectos más problemáticos y que sea capaz de conciliar el caso por caso atendiendo al interés superior del menor como principio supranacional.

Esto pasa por tener en cuenta, como ha considerado el TEDH, en relación al objeto de análisis, que lo primero a valorar por el Estado de destino en su reconocimiento previo o no de dicha filiación de menores nacidos por la técnica de la gestación por sustitución es que existe una verdadera constitución de la familia de facto y de iure, entre los padres comitentes y el menor a tenor de lo dispuesto en los principios fundamentales establecidos en el CEDH y salvaguardando los derechos fundamentales de los menores que se encuentran situados en el ámbito del DIPr de familia en una posición superior. 